\title{
A COUNTEREXAMPLE RELATED TO A CRITERION FOR A FUNCTION TO BE CONTINUOUS
}

\section{J. MICHAEL STEELE}

\begin{abstract}
A construction is given of a right-continuous function which satisfies an arithmetic continuity condition, but which is not continuous. The problem is motivated by a familiar criterion for the continuity of sample paths of a stochastic process.
\end{abstract}

In order to prove the continuity of sample functions of a stochastic process, such as Brownian motion, one sometimes applies a result like the following proposition from the forthcoming book of K. L. Chung.

Let $f$ be a real-valued function defined on $(-\infty, \infty)$ and continuous from the right everywhere. Suppose that $f$ also has left limits and satisfies

$$
\lim _{n \rightarrow \infty}\left[\max _{-\infty<k<\infty}\left|f\left(\frac{k+1}{n}\right)-f\left(\frac{k}{n}\right)\right|\right]=0 ;
$$

then $f$ continuous in $(-\infty, \infty)$.

Professor Chung recently brought to the author's attention the following question: Can one give an example which shows that the assumption of the existence of left limits cannot be dropped from the preceding proposition? There does not seem to be an example in the literature, and the arithmetical character of (1) rules out the trivial candidates. The main objective of this note is to provide a construction which settles the question and which is sufficiently systematic to prove useful in related problems.

If $C_{0}(\mathbf{R})$ denotes the class of continuous functions with compact support, one can define a new norm on $C_{0}(\mathbf{R})$ by

$$
\|f\|_{*}=\max _{p / q}\left|f\left(\frac{(p+1)}{q}\right)-f\left(\frac{p}{q}\right)\right| .
$$

The required example will be constructed as an application of a lemma which establishes a relation between $\|f\|_{*}$ and $\|f\|_{\infty}$.

LEMMA. Given any integer $M$ and $0<a<b<1$, there is a real continuous $\psi$ with support contained in $(a, b)$ for which (1) $\|\psi\|_{\infty}>M$ and (2) $\|\psi\|_{*}<5$.

Supposing this lemma for the moment, we take disjoint intervals $I_{j}=\left(a_{j}, b_{j}\right)$ with $a_{j} \nearrow 1$ and $b_{j} \nearrow 1$. By normalizing the functions provided by the lemma, we obtain functions $\phi_{j}$ such that $\operatorname{supp} \phi_{j} \subset I_{j},\left\|\phi_{j}\right\|_{\infty}=1$, and $\left\|\phi_{j}\right\|_{*}<1 / j^{2}$ for $j=1,2, \ldots$

Received by the editors March 6, 1979.

AMS (MOS) subject classifications (1970). Primary 60G05, 60G17; Secondary 20A15, $10 \mathrm{~B} 45$.

Key words and phrases. Continuous path, continuity criterion, quadratic irrational. 
On setting $f(x)=\sum_{j=1}^{\infty} \phi_{j}(x)$, one immediately sees $f(x)$ is right-continuous but not continuous; to check (1) we note

$$
\left|f\left(\frac{(k+1)}{n}\right)-f\left(\frac{k}{n}\right)\right| \leqslant \sum_{j<J}\left|\phi_{j}\left(\frac{(k+1)}{n}\right)-\phi_{j}\left(\frac{k}{n}\right)\right|+\sum_{j>J}\left\|\phi_{j}\right\|_{*} .
$$

The finite sum gives a uniformly continuous function, and the infinite sum can be made as small as we like. This completes the verification of (1) and construction of the desired example. The heart of the matter thus rests in proving the lemma we have just used.

By $F_{N}$ we denote the fractions in $[0,1]$ which have denominators no larger than $N$. We then define a step as a transition from one $p / q \in Q$ to another element of the form $p / q \pm 1 / a q \pm \varepsilon$, where $a$ is an integer and $\varepsilon$ is any rational for which $|\varepsilon|<1 / N$. Next $\psi$ is defined at each element of $F_{N} \cap(a, b)$ as the least number of steps needed to leave the interval $(a, b)$, and $\psi$ is defined to be zero at each element of $F \cap(a, b)^{c}$. The definition of $\psi$ is then extended to $\mathbf{R}$ by linear interpolation.

To see that $\|\psi\|_{*}<5$, we note $|\psi((p+1) / q)-\psi(p / q)|<1$ if $p / q \in F_{N}$ since at most one more step is needed to leave $(p+1) / q$ than was required to leave from $p / q$. Next consider $p / q \notin F_{N}$ and choose rationals $r_{1}, r_{2} \in F_{N}$ such that $\left|r_{1}-p / q\right| \leqslant 1 / N$ and $\left|r_{2}-(p+1) / q\right| \leqslant 1 / N$. Since $q>N$, one also has $\mid r_{1}-$ $r_{2} \mid<3 / N$, and consequently $\left|\psi\left(r_{1}\right)-\psi\left(r_{2}\right)\right| \leqslant 3$. The fact that $\left|\psi\left(r_{1}\right)-\psi(p / q)\right|<$ 1 and $\left|\psi\left(r_{2}\right)-\psi((p+1) / q)\right| \leqslant 1$ then completes the proof that $\|\psi\|_{*}<5$.

Now we attend to the more serious matter of showing that $N$ can be chosen so that $\|\psi\|_{\infty} \geqslant M$. To this end consider the interval $I_{0}$ consisting of the middle third of $(a, b)$. Let $l$ denote the length of $I_{0}$, and choose a quadratic irrational $\alpha \in I_{0}$. The only fact needed from diophantine analysis is that for any quadratic irrational there is a constant $c>0$ such that $|p / q-\alpha|>c / q^{2}$ for all $p / q \in Q[1, \mathrm{p} .45]$.

First take any $p_{0} / N$ such that $\left|p_{0} / N-\alpha\right|<1 / N$, and let $d_{k}$ denote the largest distance one can go from $p_{0} / N$ in $k$ steps. We then have

$$
d_{k+1} \leqslant d_{k}+\frac{1}{N}+\frac{1}{Q}
$$

where $Q$ is the smallest integer so that for some $p$ one has $\left|P / Q-p_{0} / N\right|<d_{k}$. We have $|p / Q-\alpha| \leqslant d_{k}+1 / N$ by the choice of $p_{0}$, so by bound on $|p / q-\alpha|$ mentioned above we have

$$
\frac{1}{Q}<\left(d_{k}+\frac{1}{N}\right)^{1 / 2} c^{-1 / 2}
$$

This proves the basic recursion inequality,

$$
d_{k+1}<d_{k}+\frac{1}{N}+\left(d_{k}+\frac{1}{N}\right)^{1 / 2} c^{-1 / 2}
$$

By choosing $N$ prime we can guarantee that $p_{0} / N$ is irreducible and thus $d_{1}<$ $2 / N$. The basic recursion now immediately shows that $N$ can be taken sufficiently 
large to guarantee that $d_{M+1}<l$. Since $\alpha$ is in the middle third of $(a, b)$, the inequality $d_{M+1}<l$ implies that one cannot go from $p_{0} / N$ to the exterior of $(a, b)$ in $M$ or fewer steps. This is naturally equivalent to saying $\psi\left(p_{0} / N\right)>M$, so $\|\psi\|_{\infty}>M$ as claimed.

\section{REFERENCES}

1. A. Ya. Khintchin, Continued fractions, Univ. of Chicago Press, Chicago, IIl., 1964.

Department of Statistics, Stanford University, Stanford, California 94305 